\title{
160. The Effect of ATP on Calcium Release Mechanisms in the Sarcoplasmic Reticulum of Skinned Muscle Fibers
}

\author{
By Makoto Endo and Toshio KITAzawa \\ Department of Pharmacology, Tohoku University, School of Medicine, \\ Sendai, 980, Japan \\ (Communicated by Masahiro OKadA, M. J. A., Dec. 13, 1976)
}

The sarcoplasmic reticulum (SR) of skeletal muscle releases calcium to induce contraction, and reaccumulates the ion to cause relaxation..$^{2), 8,11), 14)}$ The mechanism of calcium accumulation by the SR has been known to be essentially an ATP-dependent active transport of the ion. ${ }^{2,8), 14)}$ On the other hand, the mechanism of physiological calcium release is not yet clear, but believed to be a process entirely independent of the calcium accumulating mechanism.5) If so, calcium release may occur irrespective of the presence or absence of ATP in the medium. Indeed, calcium release by "depolarization" of the fragmented SR was shown to occur in the absence of ATP. ${ }^{9)}$ We have further examined the effects of ATP on this and other calcium release mechanisms in the SR of skinned muscle fibers.

Methods. Skinned fibers from iliofibularis muscles of African clawed toads, Xenopus laevis were used. Methods and procedures were almost the same as previously described, ${ }^{3), 6)}$ except for the following. Magnesium and calcium methanesulfonates were used in place of sulfates. In ATP-free solutions, magnesium methanesulfonate was usually reduced to $0.9 \mathrm{mM}$ to keep the same level of free magnesium. ${ }^{4}$ ) When necessary to keep free magnesium at a very low level, $10 \mathrm{mM}$ CyDTA (1,2-cyclohexanediamine-tetraacetic acid) was used.

Results. Calcium outflux from $S R$ in the absence of ATP. Since it was suggested that removal of ATP by itself may cause release of calcium from the fragmented $S R,{ }^{17)}$ the effect of removal of ATP on outflux of calcium from the SR was first studied. The SR of a skinned fiber was loaded with calcium to a fixed level, and then immersed, for various periods of time, in either a normal relaxing solution or a solution similar to it but without addition of ATP (Rigor tension was developed and maintained during the period of absence of ATP, but quickly disappeared on reintroduction of ATP). The amount of calcium in the SR was then measured by applying caffeine. ${ }^{6)}$ It slowly decreased with time of the immersion in solutions 
containing EGTA. The time course of decrease was not faster but the same, or even slower, in the absence of ATP than in its presence. Thus, the result of skinned fibers was contrary to the suggestion mentioned above, but in an essential agreement with other fragmented SR experiments. ${ }^{1)}$

"Depolarization"-induced release of calcium in the absence of ATP. The SR of skinned fibers was loaded with calcium to a fixed level, and then "depolarized" by replacing methanesulfonate with chloride $^{6)}$ either in the presence or absence of ATP. As shown in Fig. 1a, the amount of calcium released was smaller in the absence of ATP than in its presence, but it is quite clear that "depolarization" could induce calcium release in the complete absence of ATP. In some fibers, the magnitude of "depolarization"-induced release in the absence of ATP was almost the same as that in its presence.

Calcium- and caffeine-induced release of calcium in the absence of ATP. In contrast, calcium-induced calcium release was strongly inhibited in the absence of ATP. Thus, $10^{-3} \mathrm{M}$ free calcium, which showed a distinct calcium-releasing effect in the presence of ATP,3),4) did not release calcium in the absence of ATP. Caffeine-induced release, which was considered as a result of enhancement of the calcium-induced release, ${ }^{4)}$ was also very strongly inhibited in the absence of ATP, as shown in Fig. 1b. Qualitatively, however, the effect of caffeine in the absence of ATP was similar to that in its presence. Thus, for example, although $25 \mathrm{mM}$ caffeine failed to cause

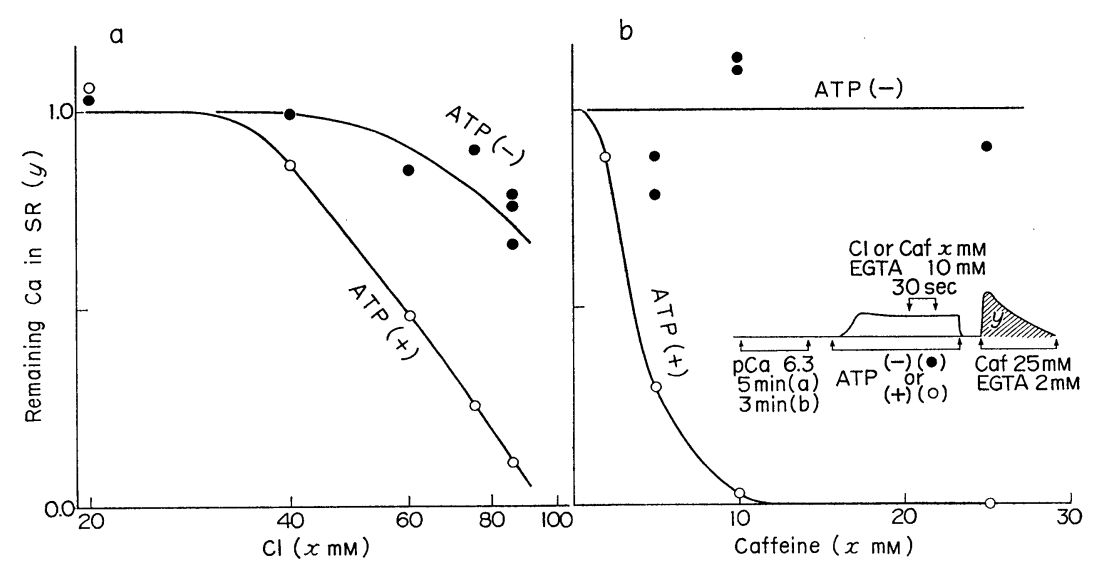

Fig. 1. Comparison of calcium release in the presence and absence of ATP. Experimental procedures were similar to those described before $^{3), 6), 13)}$, and are illustrated in the inset of the figure. The response without calcium-releasing stimulus was taken as standard for remaining calcium in the ordinate. (a) "Depolarization"-induced release. Fiber 760904. (b) Caffeine-induced release. Fiber 751025. 


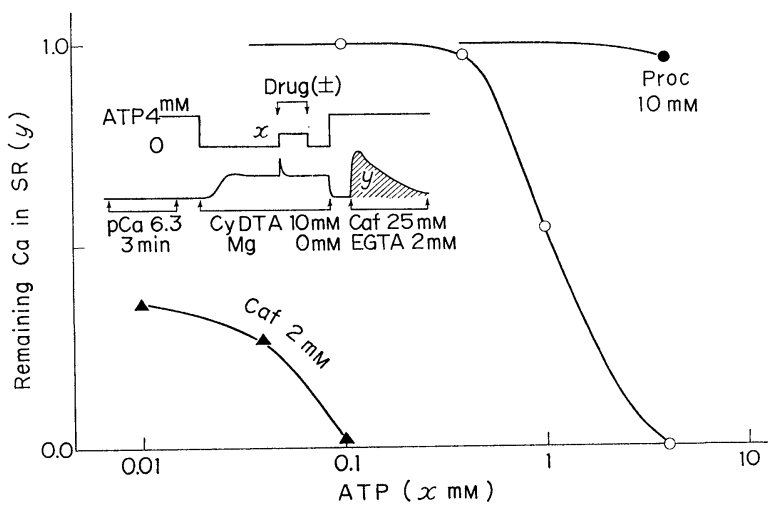

Fig. 2. The effect of ATP on calcium release under a very low level of free magnesium. Experimental procedures are illustrated in the inset of the figure. Fiber 760313. For further explanation, see text.

an appreciable release of calcium in an ATP-free solution under the condition of Fig. 1b, it induced a good release when free calcium was raised from less than $10^{-9} \mathrm{M}$ to $10^{-6} \mathrm{M}$ (cf. ref. 4), and the release was almost complete when $0.9 \mathrm{mM}$ free magnesium present was withdrawn further (cf. ref. 4). The latter releases by caffeine in the absence of ATP were completely inhibited by $10 \mathrm{mM}$ procaine (cf. ref. $7,12,13)$.

Stimulating effect of free ATP on calcium-induced calcium release mechanism. Is the enhancement of calcium release in the presence of ATP described above attributable to MgATP or to free ATP? Figure 2 shows that free ATP has the stimulant action. The loaded SR was treated with a solution in which no magnesium and $10 \mathrm{mIM}$ CyDTA was added so that free magnesium was less than $10^{-9} \mathrm{M}$. This resulted in a large release of calcium if $4 \mathrm{mM}$ ATP was present, probably because of an activation of calcium-induced calcium release mechanism, since lowering free magnesium was known to enhance the mechanism. ${ }^{3), 5)}$ However, if concentration of ATP was reduced, calcium release was inhibited as shown in Fig. 2. In this case, since the concentration of MgATP was negligible, the effect must have been exerted by free ATP. Figure 2 also shows that the effect of free ATP was inhibited by procaine, and enhanced by caffeine, as expected if the calcium release was really a result of activation of calcium-induced calcium release mechanism. ${ }^{4), 7), 13)}$

Discussion. The results reported above shows that ATP also affects calcium release processes in the SR. However, both "depolarization"9)- and caffeine- (calcium-) induced release could be evoked in the absolute absence of ATP, which is distinct from the active accumulation of calcium by the SR.2),8),14) 
[Vol. 52,

Although both kinds of calcium release are inhibited in the absence of ATP, the magnitude of inhibition was quite different; the inhibition of calcium-induced calcium release was much stronger. This might support our previous conclusion that these two kinds of stimuli are independent. ${ }^{13)}$

The above results clearly show the effect of free ATP on calcium release. This is also distinct from calcium uptake, which requires MgATP. ${ }^{2), 8), 14)}$ An effect of free ATP was also noted in the fragmented SR. ${ }^{16)}$ The present result does not exclude, however, the possibility that MgATP also has a similar action on calcium release. A similar stimulating effect of ATP on caffeine-induced calcium release in the fragmented SR was reported previously. ${ }^{17)}$

The strong dependence of calcium-induced calcium release on ATP might suggest that this release mechanism may have some relation to calcium pump mechanism. However, it is quite clear that calcium-induced release is not the reversal of the pump.5) An interesting idea is that the carrier for the calcium pump is decoupled from the ATP-splitting system under the condition and acts as a carrier for calcium-induced calcium release. ${ }^{5), 10)}$ At present, there is no clear evidence either to support or to deny this.

\section{References}

1) Carvalho, A. P., and Leo, B. (1967): J. Gen. Physiol., 50, 1327-1352.

2) Ebashi, S., and Endo, M. (1968): Progr. Biophys. Mol. Biol., 18, 123-183.

3) Endo, M. (1975) : Proc. Japan Acad., 51, 467-472.

4) - (1975) : Proc. Japan Acad., 51, 479-484.

5) - (1977): Physiol. Rev., 57(1), in press.

6) Endo, M., and Nakajima, Y. (1973): Nature (New Biol.), 246, 216-218.

7) Ford, L. E., and Podolsky, R. J. (1972) : J. Physiol., 223, 1-19.

8) Hasselbach, W. (1964): Progr. Biophys. Mol. Biol., 14, 167-222.

9) Kasai, M., and Miyamoto, H. (1976): J. Biochem., 79, 1067-1076.

10) Ogawa, Y., and Ebashi, S. (1976): J. Biochem., 80, 1149-1157.

11) Sandow, A. (1965): Pharmacol. Rev., 17, 265-320.

12) Schuller, J. (1925) : Arch. Exp. Path. Pharmakol., 105, 224-237.

13) Thorens, S., and Endo, M. (1975) : Proc. Japan Acad., 51, 473-478.

14) Weber, A. (1966): Current Topics in Bioenergetics (ed. D. R. Sanadi). Academic Press, New York and London, Vol. 1, pp. 203-254.

15) - (1968): J. Gen. Physiol., 52, 760-772.

16) - (1971): J. Gen. Physiol., 57, 50-63.

17) - (1971): J. Gen. Physiol., 57, 64-70. 